\title{
Intoxicação latrogênica de um felino por Cloridrato de Bupivacaína
}

\author{
latrogenic Intoxication of a feline by Bupivacaine Hidrochloride \\ Luciana Branquinho Queiroga ${ }^{1}$, Andrea Marques Gentili ${ }^{2}$, Ruben Lundgren Cavalcanti ${ }^{1}$, \\ Nádia Outeda Crosignani ${ }^{1}$, Alexandre da Silva Polydoro ${ }^{1} \&$ Cláudio Correa Natalini ${ }^{3}$
}

\begin{abstract}
RESUMO
O Cloridrato de Bupivacaína é um anestésico local do tipo amida de ação duradoura que, quando administrado em altas doses ou por vias inadequadas, pode acarretar efeitos tóxicos aos sistemas nervoso central (SNC) e cardiocirculatório. Este trabalho visa a relatar o quadro clínico desenvolvido por um felino, da raça siamesa, de 8 meses, do sexo feminino, intoxicado pela administração iatrogência de cloridrato de bupivacaína, trazido ao Hospital Veterinário Mundo Animal, apresentando quadro convulsivo, evoluindo para depressão do SNC e alterações cardiovasculares; bem como a terapia instituída no controle dos sinais apresentados. Foi instituída terapêutica de suporte, ventilação com oxigênio a 100\%, controle das convulsões com o uso de benzodiazepínicos e controle do edema pulmonar e efusões pleurais e pericárdicas com o uso de furosemida. O paciente evoluiu positivamente, recebendo alta após 5 dias.
\end{abstract}

Descritores: felinos, intoxicação, cloridrato de bupivacaína.

\section{ABSTRACT}

Bupivacaine hidrochloride is a long duration amida type local anesthesic. When high doses were administrated it can cause toxicity to the nervous system and cardiocirculatory system. This report describes the clinical signs of a cat intoxicated by iatrogenic administration of bupivacaine hidrochloride. The patient was brought to Hospital Veterinário Mundo Animal presenting convulsions, developing depression of central nervous system and cardiovascular changes. It was established therapeutic support, ventilation with oxygen to $100 \%$, control of seizures with the use of benzodiazepines and control of pulmonary edema and pleural and pericardic efusions with the use of furosemide. The patient has positive developments, getting high after 5 days.

Keywords: feline, intoxication, bupivacaine hidrochloride. 


\section{INTRODUÇÃO}

Anestésicos locais bloqueiam de maneira reversível a condução nervosa por meio do bloqueio de canais de sódio na membrana celular, sendo classificados como aminoésteres e aminoamidas, sendo que os últimos sofrem metabolismo hepático e excreção renal, não produzindo metabólicos tóxicos [2,4,5].

O Cloridrato de Bupivacaína é um anestésico local do tipo amida de ação duradoura que, em níveis excessivos, pode causar alterações neurológicas e cardiovasculares $[2,4,7]$. O tratamento, no caso de intoxicações, consiste em oxigenação e correção do desequilíbrio ácido-básico, medidas de suporte e controle de convulsões [2,5].

\section{RELATO DE CASO}

Uma gata siamesa de oito meses, pesando $3 \mathrm{~kg}$ foi atendida no Hospital Veterinário Mundo Animal Porto Alegre, RS, apresentando convulsões generalizadas, cianose, taquicardia e edema pulmonar, após ter sido administrado, por via intramuscular (IM), $6 \mathrm{mg} . \mathrm{kg}^{-1}$ de cloridrato de bupivacaína ${ }^{1}$. Foi administrado diazepam ${ }^{2}$, ocorrendo parada respiratória, sendo

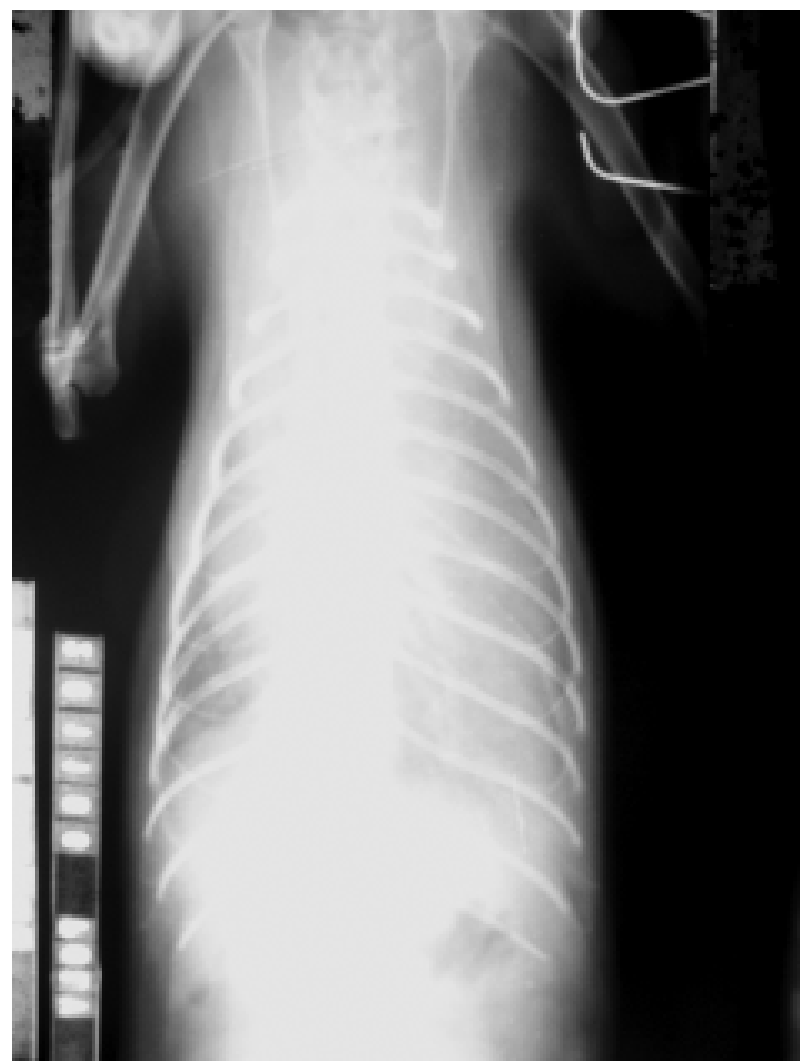

Figura 1. Radiologia torácica evidenciando efusão pelural em felino intoxicado por cloridrato de bupivacaína. realizada intubação orotraqueal e ventilação com oxigênio $\left(\mathrm{O}_{2}\right)$ a $100 \%$, seguido de fluidoterapia com solução de ringer lactato ${ }^{3}$ e administração de furosemida ${ }^{4}$. Após 2 horas, a paciente apresentou respiração espontânea e controle das convulsões.

Foi observada efusão pleural ao exame radiológico (Figura 1), sendo realizada toracocentese bilateral. Foi drenando $120 \mathrm{ml}$ de líquido serossanguinolento classificado como transudato modificado e realizado novo exame radiológico, o qual sugeriu efusão pericárdica (Figura 2). Foi mantida a administração de furosemida a cada 8 horas. Realizou-se eletrocardiografia (ECG) 72 horas após, a qual apresentou-se dentro da normalidade. Foram observadas leucopenia $\left(5500 / \mathrm{mm}^{3}\right)$ e linfopenia $\left(880 / \mathrm{mm}^{3}\right)$, além de aumento das atividades das enzimas ALT (212,10UI/L) e AST (106,30UI/L).

Após 5 dias, observou-se normalização do leucograma, trombocitopenia (plaquetas $=50.000 / \mathrm{mm}^{3}$ ), ALT (120,00UI/L) e AST (70,70 UI/L), sendo iniciada terapia com prednisolona 5 . Após 10 dias, hematologia, bioquímica sérica e radiologia apresentaram-se dentro da normalidade para a espécie.

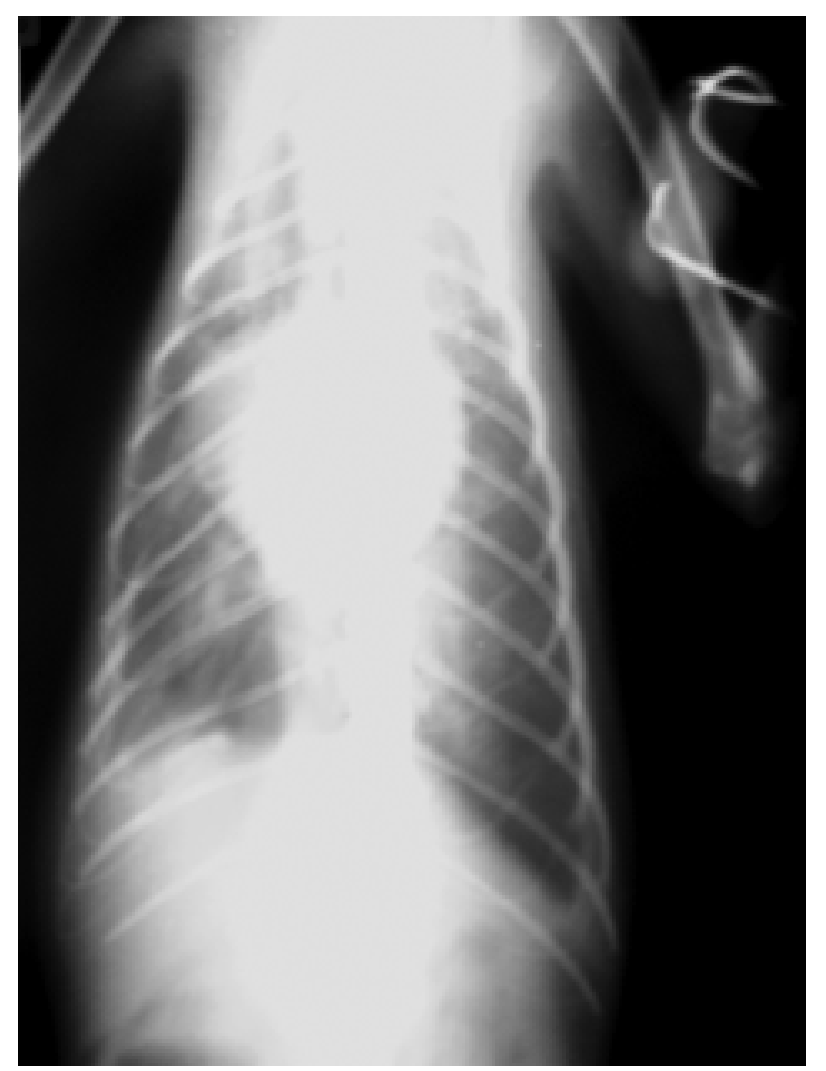

Figura 2. Radiologia torácica evidenciando efusão cardíaca após toracocentese bilateral em felino intoxicado por cloridrato de bupivacaína. 


\section{DISCUSSÃO}

Embora o uso de anestésicos locais seja seguro, a administração em dose excessiva promoveu intoxicação da paciente, levando a convulsões, depressão do SNC e lesão miocárdica, ocasionando bradicardia, edema pulmonar e efusão torácica, além de alterações das enzimas ALT e AST [2,4,6,7].

Foi instituída a terapia anticonvulsivante com benzodiazepínico indicado pela bibliografia e oxigenação, além de instituída fluidoterapia alcalinizante, visando-se a reverter os quadros de hipóxia e acidose causados por depressão respiratória e convulsões $[1,2,4,5]$. O uso de furosemida se mostrou eficiente no controle do edema pulmonar e efusões torácicas, embora não tenha seu uso relatado em intoxicações por anestésicos locais. Não foram evidenciadas alterações no ECG, provavelmente por sua realização tardia $[1,3,7]$.

É cada vez mais comum a utilização de anestésicos locais em combinação com a anestesia geral visando diminuir a dosagem de fármacos e a incidência de efeitos tóxicos. Assim, é importante que o clínico esteja capacitado para identificar, previnir e tratar tais intoxicações.

\section{NOTAS INFORMATIVAS}

'Neocaína - CRISTÁLIA - Produtos Químicos Farmacêuticos Ltda, Itapira/ SP.

${ }^{2}$ Valium - Hoffmann-La Roche Ltda, Alemanha.

${ }^{3}$ Ringer lactato - CRISTÁLIA - Produtos Químicos Farmacêuticos Ltda, Itapira/ SP.

${ }^{4}$ Lasix - Hoechst, São Paulo/SP.

${ }^{5}$ Meticortem Veterinário - Schering-Plough Saúde Animal Indústria e Comércio Ltda, Cotia/SP.

\section{REFERÊNCIAS}

1 Brown D.L. 2007. Local Anesthetic toxicity. In: Finucani B.T. Complications of Regional Anesthesia. 2n. edn. Alberta: Springer Science + Business Media, LLC, p.61-71.

2 Fantoni D.T. \& Cortopassi S.R.G. 2002. Anestesia em Cães e Gatos. São Paulo: Editora Roca, 389p.

3 Groban L., Deal D.D., Vernon J.C., James R.L. \& Butterworth L. 2000. Ventricular arrhythmias with or without programmed eletrical stimulation after incremental overdosage with lidocaine, bupivcaine, levobupivacaine and ropivacaine. Anesthesia and Analgesia. 91: 1103-1111.

4 Natalini C.C. 2007. Anestesias local e regional. In: Teorias e Técnicas em Anestesiologia Veterinária. Porto Alegre: Artmed, p.205-229.

5 Stoelting R.K. \& Hiller S.C. 2007. Anestésicos Locais. In: Farmacología e Fisiologia na Prática Anestésica. 2. ed. Porto Alegre: Artmed, pp.159-187.

6 Tilley L.P. \& Smith Jr. F.W.K. 2003. Consulta Veterinária em 5 Minutos Espécies Canina e Felina. 2. ed. São Paulo: Manole, 1423p.

7 Veering B.T. 2003. Complications and local anesthesic toxicity in regional anesthesia. Current Opinion in Aanesthesiology. 16: $455-459$. 